\title{
Supplementary file to
}

\section{"Spline-Lasso in High-Dimensional Linear Regression"}

Jianhua GUO, Jianchang HU, Bing-Yi JING, Zhen ZHANG

NENU, HKUST, HKUST, NUS

In this supplementary file, we will give more detailed proofs to Theorems 1-3. The proof of Theorem 1 follows similar lines to those in Hebiri and Van de Geer (2011) while the proofs of Theorems 2-3 follow similar lines to those in Huang et al. (2011).

\section{Proof of Theorem 1}

We first give a lemma on a concentration result dealing with Gaussian noise:

Lemma 1. Let $\eta \in(0,1)$. Denote $\Lambda_{n, p}=\left\{\max _{j=1, \ldots, p} 2\left|V_{j}\right| \leq \lambda_{1} / 2\right\}$ where $V_{j}=n^{-1} \sum_{i=1}^{n} x_{i, j} \varepsilon_{i}$. Let $\lambda_{1}$ be defined as in Theorem 1. Then

$$
P\left(\max _{j=1, \ldots, p} 2\left|V_{j}\right| \leq \lambda_{1} / 2\right) \geq 1-\eta
$$


The second lemma states that the squared risk and the $l_{1}$ estimation error can be controlled by the restricted $l_{2}$ estimation error $\left\|\beta_{\mathcal{B}}-\hat{\beta}_{\mathcal{B}}^{\text {spline }}\right\|_{2}$.

Lemma 2. On the event $\Lambda_{n, p}=\left\{\max _{j=1, \ldots, p} 2\left|V_{j}\right| \leq \lambda_{1} / 2\right\}$, we have

$$
\frac{1}{n}\left\|\tilde{X} \beta-\tilde{X} \hat{\beta}^{\text {spline }}\right\|_{2}^{2}+\frac{\lambda_{1}}{2}\left\|\beta-\hat{\beta}^{\text {spline }}\right\|_{1} \leq r_{n}\left\|\beta_{\mathcal{B}}-\hat{\beta}_{\mathcal{B}}^{\text {spline }}\right\|_{2},
$$

where $\tilde{X}=\left(X, \sqrt{n \lambda_{2}} L\right)^{T}, r_{n}=2 \lambda_{1} \sqrt{|\mathcal{A}|}+2 \lambda_{2}\|\tilde{J} \beta\|_{2}$, and $\mathcal{B} \supseteq \mathcal{A}$.

Proof. Consider the augmented data set:

$$
\tilde{X}=\left(X, \sqrt{n \lambda_{2}} L\right)^{T} \text {, and } \tilde{Y}=(Y, \mathbf{0})^{T} \text {, and } \tilde{\varepsilon}=\left(\varepsilon,-\sqrt{n \lambda_{2}} L \beta\right)^{T} \text {. }
$$

Then the spline-lasso estimator is the minimizer of $n^{-1}\|\tilde{Y}-\tilde{X} \beta\|_{2}^{2}+\lambda_{1}\|\beta\|_{1}$. Therefore, we have

$$
\begin{aligned}
& n^{-1}\left\|\tilde{Y}-\tilde{X} \hat{\beta}^{\text {spline }}\right\|_{2}^{2}+\lambda_{1}\left\|\hat{\beta}^{\text {spline }}\right\|_{1} \leq n^{-1}\|\tilde{Y}-\tilde{X} \beta\|_{2}^{2}+\lambda_{1}\|\beta\|_{1}, \\
& \text { i.e., } \\
& \frac{1}{n}\left\|\tilde{X} \beta-\tilde{X} \hat{\beta}^{\text {spline }}\right\|_{2}^{2} \leq \lambda_{1}\left(\|\beta\|_{1}-\left\|\hat{\beta}^{\text {spline }}\right\|_{1}\right)+\frac{2}{n} \tilde{\varepsilon}^{T} \tilde{X}\left(\beta-\hat{\beta}^{\text {spline }}\right) .
\end{aligned}
$$

Then on the event $\Lambda_{n, p}=\left\{\max _{j=1, \ldots, p} 2\left|V_{j}\right| \leq \lambda_{1} / 2\right\}$, we have

$$
\begin{aligned}
\frac{1}{n}\left\|\tilde{X} \beta-\tilde{X} \hat{\beta}^{\text {spline }}\right\|_{2}^{2} \leq & \lambda_{1}\left(\|\beta\|_{1}-\left\|\hat{\beta}^{\text {spline }}\right\|_{1}\right)+\frac{\lambda_{1}}{2}\left\|\beta-\hat{\beta}^{\text {spline }}\right\|_{1} \\
& -2 \lambda_{2} \beta^{T} \tilde{J}\left(\beta-\hat{\beta}^{\text {spline }}\right),
\end{aligned}
$$

where $\tilde{J}=L^{T} L$. Then consider a set $\mathcal{B}$ such that $\left\|\beta_{\mathcal{B}}-\hat{\beta}_{\mathcal{B}}^{\text {spline }}\right\|_{2}=\| \beta-$ $\hat{\beta}^{\text {spline }} \|_{2}$. Such a set exists, for instance, $\mathcal{B}=\left\{j \in\{1, \ldots, p\}: \hat{\beta}^{\text {spline }}=\right.$ 0 and $\beta=0\}^{C}$, and it includes $\mathcal{A}$. Then we have

$$
-\lambda_{2} \beta^{T} \tilde{J}\left(\beta-\hat{\beta}^{\text {spline }}\right) \leq \lambda_{2}\|\tilde{J} \beta\|_{2}\left\|\beta_{\mathcal{B}}-\hat{\beta}_{\mathcal{B}}^{\text {spline }}\right\|_{2}
$$


Hence, we have

$$
\begin{aligned}
& \frac{1}{n}\left\|\tilde{X} \beta-\tilde{X} \hat{\beta}^{\text {spline }}\right\|_{2}^{2}+\frac{\lambda_{1}}{2}\left\|\beta-\hat{\beta}^{\text {spline }}\right\|_{1} \\
\leq & \lambda_{1}\left[\|\beta\|_{1}-\left\|\hat{\beta}^{\text {spline }}\right\|_{1}+\left\|\beta-\hat{\beta}^{\text {spline }}\right\|_{1}\right]+2 \lambda_{2}\|\tilde{J} \beta\|_{2}\left\|\beta_{\mathcal{B}}-\hat{\beta}_{\mathcal{B}}^{\text {spline }}\right\|_{2} \\
\leq & 2 \lambda_{1} \sum_{j \in \mathcal{A}}\left\|\beta_{j}-\hat{\beta}_{j}^{\text {spline }}\right\|+2 \lambda_{2}\|\tilde{J} \beta\|_{2}\left\|\beta_{\mathcal{B}}-\hat{\beta}_{\mathcal{B}}^{\text {spline }}\right\|_{2} \\
\leq & r_{n}\left\|\beta_{\mathcal{B}}-\hat{\beta}_{\mathcal{B}}^{\text {spline }}\right\|_{2},
\end{aligned}
$$

where $r_{n}=2 \lambda_{1} \sqrt{|\mathcal{A}|}+2 \lambda_{2}\|\tilde{J} \beta\|_{2}$ and this proves the lemma.

Returning to the proof of Theorem 1 , from the above lemmas, we have

$$
\left\|\beta-\hat{\beta}^{\text {spline }}\right\|_{1} \leq \varrho_{n}\left\|\beta_{\mathcal{B}}-\hat{\beta}_{\mathcal{B}}^{\text {spline }}\right\|_{2}
$$

where $\varrho_{n}=2 r_{n} / \lambda_{1}=4 \sqrt{|\mathcal{A}|}+4 \lambda_{2} \lambda_{1}^{-1}\|\tilde{J} \beta\|_{2}$. Then the vector $\beta-\hat{\beta}^{\text {spline }}$ satisfies Assumption 1. Therefore, we get

$$
\frac{1}{n}\left\|\tilde{X} \beta-\tilde{X} \hat{\beta}^{\text {spline }}\right\|_{2}^{2} \leq \frac{r_{n}}{\sqrt{\phi_{\lambda_{2}}}} \sqrt{\frac{1}{n}}\left\|\tilde{X} \beta-\tilde{X} \hat{\beta}^{\text {spline }}\right\|_{2},
$$

which will easily lead to the first and the third part of the result

$$
\frac{1}{n}\left\|X \beta-X \hat{\beta}^{\text {spline }}\right\|_{2}^{2} \leq \phi_{\lambda_{2}}^{-1}\left(2 \lambda_{1} \sqrt{|\mathcal{A}|}+2 \lambda_{2}\|\tilde{J} \beta\|_{2}\right)^{2},
$$

and when $\lambda_{2} \neq 0$,

$$
\left\|L\left(\beta-\hat{\beta}^{\text {spline }}\right)\right\|_{2} \leq \lambda_{2}^{-1 / 2} \phi_{\lambda_{2}}^{-1 / 2}\left(2 \lambda_{1} \sqrt{|\mathcal{A}|}+2 \lambda_{2}|\tilde{J} \beta|_{2}\right) .
$$

Then the first part, together with Assumption 1, yields the second inequality.

\section{Proof of Theorem 2}

Let $\tilde{X}=\sqrt{n}\left(\Sigma+\lambda_{2} \tilde{J}\right)^{1 / 2}, \tilde{Y}=\tilde{X}^{-1} X^{T} Y$ and

$$
\tilde{M}(\mathbf{b} ; \lambda, \gamma)=(2 n)^{-1}\|\tilde{Y}-\tilde{X} \mathbf{b}\|_{2}^{2}+\sum_{j=1}^{p} \rho\left(\left|b_{j}\right| ; \lambda_{1}, \gamma\right) .
$$


Thus, $\hat{\beta}$ is also the minimizer of $\tilde{M}(\mathbf{b} ; \lambda, \gamma)$.

In order to have $\hat{\beta}=\hat{\beta}^{o}$ with high probability, $\hat{\beta}^{o}$ should satisfy the KKT conditions of $\tilde{M}$. Therefore, $\hat{\beta}_{\mathcal{O}^{\circ}}$ and $\hat{\beta}_{\mathcal{O}^{c}}^{o}$ should satisfy the KKT conditions of $\tilde{M}$ for nonzero coefficients and for zero coefficients, respectively.

It is easy to see that $\hat{\beta}_{\mathcal{O}^{c}}$ satisfies the KKT condition of $\tilde{M}$ for zero coefficients on

$$
\Omega_{1}=\left\{\left\|\tilde{X}_{\mathcal{O}^{c}}^{T}\left(\tilde{Y}-\tilde{X} \hat{\beta}^{o} / n\right)\right\|_{\infty} \leq \lambda_{1}\right\}
$$

In addition, since $\hat{\beta}_{\mathcal{O}}^{o}$ is the solution for minimizing $(2 n)^{-1}\|\tilde{Y}-\tilde{X} \mathbf{b}\|_{2}^{2}$ on the set $\mathcal{O}$ without the penalty term. Therefore, we need to have $\dot{\rho}\left(\left|\hat{\beta}_{j}^{o}\right| ; \lambda_{1}, \gamma\right)=$ $0, j \in \mathcal{O}$. Since $\left|\hat{\beta}_{j}^{o}\right| \geq \gamma \lambda_{1}$ gives $\dot{\rho}\left(\left|\hat{\beta}_{j}^{o}\right| ; \lambda_{1}, \gamma\right)=0$, so $\hat{\beta}_{\mathcal{O}^{\circ}}$ would satisfy the KKT condition of $\tilde{M}$ for nonzero coefficients on the event

$$
\Omega_{2}=\left\{\min _{j \in \mathcal{O}} \operatorname{sgn}\left(\beta_{j}^{*}\right) \hat{\beta}_{j}^{o} \geq \gamma \lambda_{1}\right\}
$$

To prove Theorem 2, it suffices to show $P\left(\Omega_{1}^{c} \cup \Omega_{2}^{c}\right) \leq 3 \epsilon$.

By $P\left(\Omega_{1}^{c} \cup \Omega_{2}^{c}\right) \leq P\left(\Omega_{1}^{c}\right)+P\left(\Omega_{2}^{c}\right)$, we try to find larger events than $\Omega_{1}^{c}$ and $\Omega_{2}^{c}$ with small probability.

Note $\Omega_{1}$ is related to the gradient in the linear model $\tilde{Y}=\tilde{X} \beta^{*}+\tilde{\varepsilon}^{*}$. Let $\tilde{\varepsilon}^{*}=\tilde{\varepsilon}+E \tilde{\varepsilon}^{*}$ with $\tilde{\varepsilon}=\tilde{Y}-E \tilde{Y}$. Since $\tilde{X}^{T} \tilde{Y}=X^{T} Y$ and both true coefficient $\beta^{o}$ and $\beta^{*}$ are supported in $\mathcal{O}$, so for any subset $B$ of indices $\{1, \ldots, p\}$,

$$
\begin{aligned}
\tilde{X}_{B}^{T} E \tilde{\varepsilon}^{*} / n & =\tilde{X}_{B}^{T}\left(E \tilde{Y}-\tilde{X} \beta^{*}\right) / n \\
& =E\left(X_{B}^{T} Y\right) / n-\tilde{X}_{B}^{T} \tilde{X} \beta^{*} / n \\
& =X_{B}^{T} X \beta^{o} / n-\tilde{X}_{B}^{T} \tilde{X}_{\mathcal{O}} \beta_{\mathcal{O}}^{*} / n \\
& =\Sigma_{B, \mathcal{O}} \beta_{\mathcal{O}}^{o}-\Sigma_{B, \mathcal{O}}\left(\lambda_{2}\right) \Sigma_{\mathcal{O}}^{-1}\left(\lambda_{2}\right) \Sigma_{\mathcal{O}} \beta_{\mathcal{O}}^{o} \\
& =\lambda_{2}\left\{\Sigma_{B, \mathcal{O}}\left(\lambda_{2}\right) \Sigma_{\mathcal{O}}^{-1}\left(\lambda_{2}\right) \tilde{J}_{\mathcal{O}}-\tilde{J}_{B, \mathcal{O}}\right\} \beta_{\mathcal{O}}^{o}
\end{aligned}
$$


Since $\tilde{X}_{\mathcal{O}}^{T} E \tilde{\varepsilon}^{*} / n=0$, we have $\left\|\tilde{X}^{T} E \tilde{\varepsilon}^{*} / n\right\|_{\infty}=\lambda_{2} C_{2}$. Since $\tilde{X}^{T} \tilde{\varepsilon}=\tilde{X}^{T} \tilde{Y}-$ $E \tilde{X}^{T} \tilde{Y}=X^{T} Y-E X^{T} Y=X^{T} \varepsilon$, we have

$$
\Omega_{1} \supseteq\left\{\left\|X_{\mathcal{O}^{c}}^{T} \varepsilon / n\right\|_{\infty}<\lambda_{1}-\lambda_{2} C_{2}\right\} .
$$

If the event on the right-hand side holds, we have

$$
\begin{aligned}
\left\|\tilde{X}_{\mathcal{O}^{c}}^{T}\left(\tilde{Y}-\tilde{X} \hat{\beta}^{o}\right) / n\right\|_{\infty} & =\left\|\tilde{X}_{\mathcal{O}^{c}}^{T}\left(\tilde{Y}-E \tilde{Y}+E \tilde{Y}-\tilde{X} \hat{\beta}^{o}\right) / n\right\|_{\infty} \\
& =\left\|\tilde{X}_{\mathcal{O}^{c}}^{T}\left(\tilde{\varepsilon}+E \tilde{\varepsilon}^{*}+\tilde{X}\left(\hat{\beta}^{o}-\beta^{*}\right)\right) / n\right\|_{\infty} \\
& =\left\|\tilde{X}_{\mathcal{O}^{c}}^{T}\left(\tilde{\varepsilon}+E \tilde{\varepsilon}^{*}\right) / n+\tilde{X}_{\mathcal{O}^{c}}^{T} \tilde{X}_{\mathcal{O}}\left(\hat{\beta}_{\mathcal{O}}^{o}-\beta_{\mathcal{O}}^{*}\right) / n\right\|_{\infty} \\
& =\left\|X_{\mathcal{O}^{c}}^{T} \varepsilon / n+\tilde{X}_{\mathcal{O}^{c}}^{T} E \tilde{\varepsilon}^{*} / n+\tilde{X}_{\mathcal{O}^{c}}^{T} \tilde{X}_{\mathcal{O}^{\prime}} \Sigma_{\mathcal{O}}^{-1}\left(\lambda_{2}\right) X_{\mathcal{O}}^{T}(Y-E Y) / n^{2}\right\|_{\infty} \\
& =\left\|X_{\mathcal{O}^{c}}^{T} \varepsilon / n+\tilde{X}_{\mathcal{O}^{c}}^{T} E \tilde{\varepsilon}^{*} / n+\tilde{X}_{\mathcal{O}^{c}}^{T} \tilde{X}_{\mathcal{O}^{\prime}} \Sigma_{\mathcal{O}}^{-1}\left(\lambda_{2}\right) X_{\mathcal{O}}^{T} \varepsilon / n^{2}\right\|_{\infty} \\
& \leq\left\|X_{\mathcal{O}^{c}}^{T} \varepsilon / n\right\|_{\infty}+\left\|\tilde{X}_{\mathcal{O}^{c}}^{T} E \tilde{\varepsilon}^{*} / n\right\|_{\infty} \leq \lambda_{1} .
\end{aligned}
$$

Since $\beta^{*}=E \hat{\beta}^{o}, \hat{\beta}_{\mathcal{O}}^{o}=\Sigma_{\mathcal{O}}^{-1}\left(\lambda_{2}\right) X_{\mathcal{O}}^{T} Y / n$ can be written as $\beta_{\mathcal{O}}^{*}+\left(\left(v_{j} / n\right)^{1 / 2} \mathbf{u}_{j}^{T} \varepsilon\right.$, $j \in \mathcal{O})^{T}$, where $\left\|\mathbf{u}_{j}\right\|_{2}=1$ and $\left\{v_{j}, j \in \mathcal{O}\right\}$ are the diagonal elements of $\Sigma_{\mathcal{O}}^{-1}\left(\lambda_{2}\right) \Sigma_{\mathcal{O}} \Sigma_{\mathcal{O}}^{-1}\left(\lambda_{2}\right)$. Thus,

$$
\Omega_{2}^{c} \subseteq \bigcup_{j \in \mathcal{O}}\left\{\operatorname{sgn}\left(\beta_{j}^{*}\right) \mathbf{u}_{j}^{T} \varepsilon \geq\left(n / v_{j}\right)^{1 / 2}\left|\beta_{j}^{*}\right| \geq \sigma \sqrt{2 \log (|\mathcal{O}| / \epsilon)}\right\} .
$$

Since $\lambda_{1} \geq \lambda_{2} C_{2}+\sigma \sqrt{2 \log \left(\left(p-d^{o}\right) / \epsilon\right)} \max _{j \leq p}\left\|\mathbf{x}_{j}\right\|_{2} / n$, the sub-Gaussian Condition yields

$$
\begin{aligned}
P\left(\Omega_{1}^{c}\right)+P\left(\Omega_{2}^{c}\right) \leq & P\left(\left\|X_{\mathcal{O}^{c}}^{T} \varepsilon / n\right\|_{\infty}>\sigma \sqrt{2 \log \left(\left(p-d^{o}\right) / \epsilon\right)} \max _{j \leq p}\left\|\mathbf{x}_{j}\right\|_{2} / n\right) \\
& +\sum_{j \in \mathcal{O}} P\left(\operatorname{sgn}\left(\beta_{j}^{*}\right) \mathbf{u}_{j}^{T} \varepsilon \geq \sigma \sqrt{2 \log (|\mathcal{O}| / \epsilon)}\right) \\
\leq & 2\left|\mathcal{O}^{c}\right| \epsilon /(p-|\mathcal{O}|)+|\mathcal{O}| \epsilon /|\mathcal{O}|=3 \epsilon .
\end{aligned}
$$




\section{Proof of Theorem 3}

$\hat{\beta}(\lambda)$ is the $\mathrm{MC}+$ estimator based on data $(\tilde{X}, \tilde{Y})$ at penalty level $\lambda_{1}$. From the proof of Theorem 5 in Zhang $(2010), \hat{\beta}(\lambda)=\hat{\beta}^{o}(\lambda)$ on $\Omega=\cap_{j=1}^{3} \Omega_{j}$, where

$$
\begin{gathered}
\Omega_{1}=\left\{\left\|\tilde{X}_{\mathcal{O}^{c}}^{T}\left(\tilde{Y}-\tilde{X} \hat{\beta}^{o} / n\right)\right\|_{\infty} \leq \lambda_{1}\right\}, \\
\Omega_{2}=\left\{\min _{j \in \mathcal{O}} \operatorname{sgn}\left(\beta_{j}^{*}\right) \hat{\beta}_{j}^{o} \geq \gamma\left(2 \sqrt{c^{*}} \lambda_{1}\right)\right\}, \\
\Omega_{3}=\left\{\zeta\left(\tilde{Y}-\tilde{\beta}^{*} ; d^{*}-|\mathcal{O}|, \mathcal{O}, \lambda_{2}\right) \leq \lambda_{1}\right\},
\end{gathered}
$$

where for $m \geq 1$ and vector $\mathbf{v}$ in the range of $\tilde{X}$,

$\zeta\left(\mathbf{v} ; m, \mathcal{O}, \lambda_{2}\right)=\max _{B}\left\{\frac{\left\|\left(\tilde{P}_{B}-\tilde{P}_{\mathcal{O}}\right) \mathbf{v}\right\|_{2}}{(m n)^{1 / 2}}: \mathcal{O} \subseteq B \subseteq\{1, \ldots, p\},|B|=m+|\mathcal{O}|\right\}$

and $\tilde{P}_{B}=\tilde{X}_{B}\left(\tilde{X}_{B}^{T} \tilde{X}_{B}\right)^{-1} \tilde{X}_{B}^{T}$.

Similar to Theorem 2, we have $\Omega_{1} \supseteq\left\{\left\|X_{\mathcal{O}^{c}}^{T} \varepsilon / n\right\|_{\infty}<\lambda_{1}-\lambda_{2} C_{2}\right\}$.

In addition, a slight modification on the proof of Theorem 2 leads to

$$
\Omega_{2}^{c} \subseteq \bigcup_{j \in \mathcal{O}}\left\{\operatorname{sgn}\left(\beta_{j}^{*}\right) \mathbf{u}_{j}^{T} \varepsilon \geq\left(n / v_{j}\right)^{1 / 2}\left(\left|\beta_{j}^{*}\right|-\gamma\left(2 \sqrt{c^{*}} \lambda_{1}\right)\right) \geq \sigma \sqrt{2 \log (|\mathcal{O}| / \epsilon)}\right\} .
$$

Then for $|B| \leq d^{*}$, we have $\left\|\tilde{P}_{B} E \tilde{\varepsilon}^{*}\right\|_{2} / \sqrt{n}=\left\|\Sigma_{B}^{-1 / 2}\left(\lambda_{2}\right) \tilde{X}_{B}^{T} E \tilde{\varepsilon}^{*}\right\|_{2} / n \leq$ $\left\|\tilde{X}_{B}^{T} E \tilde{\varepsilon}^{*} / n\right\|_{\infty} \sqrt{|B| / c_{*}\left(\lambda_{2}\right)}$, and

$$
\left\|\tilde{P}_{B} \tilde{\varepsilon}\right\|_{2} / \sqrt{n}=\left\|\Sigma_{B}^{-1 / 2}\left(\lambda_{2}\right) \tilde{X}_{B}^{T} \tilde{\varepsilon}\right\|_{2} / n \leq\left\|X_{B}^{T} \varepsilon / n\right\|_{\infty} \sqrt{|B| / c_{*}\left(\lambda_{2}\right)} .
$$

Thus,

$$
\begin{aligned}
& \zeta\left(\tilde{Y}-\tilde{\beta}^{*} ; d^{*}-|\mathcal{O}|, \mathcal{O}, \lambda_{2}\right) \\
= & \zeta\left(\tilde{\varepsilon}+E \tilde{\varepsilon}^{*} ; d^{*}-|\mathcal{O}|, \mathcal{O}, \lambda_{2}\right) \\
= & \max \left\{\frac{\|\left.\left(\tilde{P}_{B}-\tilde{P}_{\mathcal{O}}\right)\left(\tilde{\varepsilon}+E \tilde{\varepsilon}^{*}\right)\right|_{2}}{\left(\left(d^{*}-|\mathcal{O}|\right) n\right)^{1 / 2}}: \mathcal{O} \subseteq B \subseteq\{1, \ldots, p\},|B|=d^{*}\right\}
\end{aligned}
$$




$$
\begin{aligned}
& \leq \frac{\max _{B}\left\{\left\|X_{B}^{T} \varepsilon / n\right\|_{\infty} \sqrt{|B| / c_{*}\left(\lambda_{2}\right)}+\left\|\tilde{X}_{B}^{T} E \tilde{\varepsilon}^{*} / n\right\|_{\infty} \sqrt{|B| / c_{*}\left(\lambda_{2}\right)}\right\}}{\left(d^{*}-|\mathcal{O}|\right)^{1 / 2}} \\
& \leq \frac{\left(|| X^{T} \varepsilon / n \|_{\infty}+\lambda_{2} C_{2}\right) \sqrt{d^{*}}}{\sqrt{\left(d^{*}-|\mathcal{O}|\right) c_{*}\left(\lambda_{2}\right)}} .
\end{aligned}
$$

Since $|\mathcal{O}| \leq d^{*} /\left(K_{*}+1\right)$, this gives $\Omega_{3} \supseteq\left\{\left\|X^{T} \varepsilon / n\right\|_{\infty}<\sqrt{c_{*}\left(\lambda_{2}\right) K_{*} /\left(K_{*}+1\right)} \lambda_{1}-\right.$ $\left.\lambda_{2} C_{2}\right\}$. Therefore,

$$
\begin{aligned}
1-P\left\{\Omega_{1} \cap \Omega_{3}\right\}+P\left\{\Omega_{2}^{c}\right\} \leq & P\left(\left\|X^{T} \varepsilon / n\right\|_{\infty}>\sigma \sqrt{2 \log (p / \epsilon)} \max _{j \leq p}\left\|\mathbf{x}_{j}\right\|_{2} / n\right) \\
& +\sum_{j \in \mathcal{O}} P\left(\operatorname{sgn}\left(\beta_{j}^{*}\right) \mathbf{u}_{j}^{T} \varepsilon \geq \sigma \sqrt{2 \log (|\mathcal{O}| / \epsilon)}\right) \\
\leq & 2 p(\epsilon / p)+|\mathcal{O}| \epsilon /|\mathcal{O}|=3 \epsilon .
\end{aligned}
$$

This completes the proof, since $\hat{\beta}_{j}^{o} \neq 0$ for all $j \in \mathcal{O}$ in $\Omega_{2}$. 